\title{
Principles and Practice of HREM and HAADF Imaging
}

\author{
B.G.Mendis*
}

* Dept of Physics, Durham University, South Road, Durham, DH1 3LE, UK

High resolution electron microscopy (HREM) and high angle annular dark field (HAADF) are two of the most widely used methods for imaging the atomic structure of a material. In HREM the unscattered and diffracted beams are used to form the image [1-2]. Interference of the electron beams depend on several parameters, such as strain, orientation and lens aberrations. Furthermore the relative intensities of the beams vary as a function of specimen thickness, making HREM images sensitive to dynamic scattering. HAADF images, on the other hand, are acquired in scanning transmission mode by collecting the high angle scattered intensity at each probe position [3-4]. HAADF images are incoherent, so that they do not suffer from 'contrast reversals' due to varying specimen thickness and defocus.

The recent development of aberration correction has seen an explosion in the use of HREM and HAADF imaging to solve complex materials problems. Aberration correctors vary from quadrupoleoctupole designs used in dedicated STEMs [5-6] to the long hexapoles used in TEM/STEM instruments [7-8]. In HREM further computer processing techniques such as exit-wave reconstruction can be used to remove the effects of residual aberrations [9]. HAADF imaging can also be combined with electron energy loss spectroscopy to give structural and chemical information at the atomic scale [10], thereby extracting maximum information from the specimen.

The current resolution limits achieved in HREM and HAADF through aberration correction is adequate for most materials characterisation work. However, since HREM and HAADF images are recorded in transmission the $3 \mathrm{D}$ information from the sample is lost. This is a weakness compared to techniques such as atom probe tomography. The large objective aperture size in an aberration corrected STEM microscope enables optical sectioning of the specimen, so that (for example) the depth distribution of dopant atoms within a semiconductor can be determined [11], although the outof-plane resolution is still far inferior to the in-plane resolution. Furthermore in the HREM examination of end-on dislocations surface relaxation of the thin foil takes place. The surface relaxation is largest for screw type dislocations and is known as the Eshelby twist. The Eshelby twist for isotropic materials is equal and opposite at the two free surfaces, so that the net distortion of an atom column, integrated through the thickness of the foil, is zero. However, the electron beam scattering is such that a residual twist displacement is still observed in the final HREM image [12]. As an example the HREM image for an end-on, 1/2[111] screw dislocation in a $5 \mathrm{~nm}$ thick Mo foil containing the Eshelby twist was simulated using the multislice method. Atom displacements due to the strain field of the screw dislocation are along the specimen thickness direction and therefore cannot be observed. The imaging conditions were such that the intensity maxima in the HREM image correspond to the atom column positions. The displacement of the intensity maxima, with respect to the atom column positions in an equivalent crystal containing no Eshelby twist, is shown in Fig.1. Large displacements which have the same form of the Eshelby twist are observed. Surface relaxation therefore has important implications for structural analysis of dislocations through projected HREM images. More work on electron beam scattering mechanisms is required if the projection problem is to be overcome or minimised in such cases [13]. 


\section{References}

[1] J.C.H. Spence, High-Resolution Electron Microscopy, Oxford University Press, 2003.

[2] P.R. Buseck et al., High-Resolution Transmission Electron Microscopy and Associated Techniques, Oxford University Press, 1988.

[3] S.J. Pennycook, D.E. Jesson, Ultramicroscopy 37 (1991) 14.

[4] P. D. Nellist, S. J. Pennycook, Ultramicroscopy 78 (1999) 111.

[5] O.L.Krivanek et al., Ultramicroscopy 78 (1999) 1.

[6] O.L. Krivanek et al., Ultramicroscopy 108 (2008) 179.

[7] M. Haider et al., Ultramicroscopy 75 (1998) 53.

[8] J.L. Hutchison et al., Ultramicroscopy 103 (2005) 7.

[9] L.Y. Chang, A.I. Krikland, Microsc. Microanal. 12 (2006) 469.

[10] M. Varela et al., Phys. Rev. Lett. 92 (2004) 095502.

[11] J.E. Allen et al., Nature Nanotech. 3 (2008) 168.

[12] B.G. Mendis et al., Phil. Mag. 86 (2006) 4607.

[13] B.G. Mendis, K.J. Hemker, Ultramicroscopy 108 (2008) 855.

[14] BGM would like to thank the Dept. of Physics, Durham University for financial support.

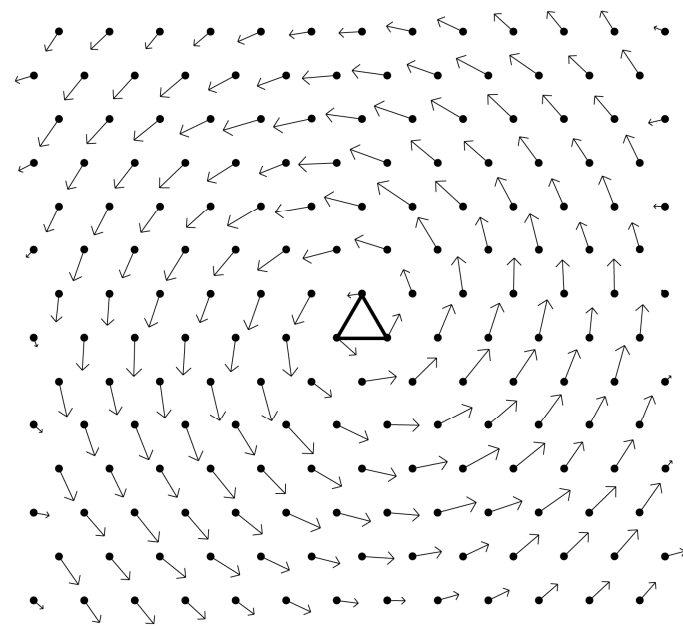

FIG. 1. Displacements of intensity maxima for a multislice simulated, HREM image of an end-on, $1 / 2[111]$ screw dislocation in a $5 \mathrm{~nm}$ thick Mo foil containing the Eshelby twist. The displacement vectors are determined with respect to an equivalent crystal free of the Eshelby twist and are magnified by $\mathrm{x} 15$ for visual clarity. The dislocation is at the centroid of the triangle superimposed in the figure. 